University of Warwick institutional repository: http://go.warwick.ac.uk/wrap This paper is made available online in accordance with publisher policies. Please scroll down to view the document itself. Please refer to the repository record for this item and our policy information available from the repository home page for further information.

To see the final version of this paper please visit the publisher's website. Access to the published version may require a subscription.

Author(s): A. FLETCHER

Article Title: LOCAL RIGIDITY OF INFINITE-DIMENSIONAL TEICHMÜLLER SPACES

Year of publication: 2006

Link to published version:

http://dx.doi.org/10.1112/S0024610706023003

Publisher statement: None 


\title{
LOCAL RIGIDITY OF INFINITE-DIMENSIONAL TEICHMÜLLER SPACES
}

\author{
A. FLETCHER
}

\begin{abstract}
This paper presents a rigidity theorem for infinite-dimensional Bergman spaces of hyperbolic Riemann surfaces, which states that the Bergman space $A^{1}(M)$, for such a Riemann surface $M$, is isomorphic to the Banach space of summable sequence, $l^{1}$. This implies that whenever $M$ and $N$ are Riemann surfaces that are not analytically finite, and in particular are not necessarily homeomorphic, then $A^{1}(M)$ is isomorphic to $A^{1}(N)$. It is known from $\mathrm{V}$. Markovic that if there is a linear isometry between $A^{1}(M)$ and $A^{1}(N)$, for two Riemann surfaces $M$ and $N$ of nonexceptional type, then this isometry is induced by a conformal mapping between $M$ and $N$. As a corollary to this rigidity theorem presented here, taking the Banach duals of $A^{1}(M)$ and $l^{1}$ shows that the space of holomorphic quadratic differentials on $M, Q(M)$, is isomorphic to the Banach space of bounded sequences, $l^{\infty}$. As a consequence of this theorem and the Bers embedding, the Teichmüller spaces of such Riemann surfaces are locally bi-Lipschitz equivalent.
\end{abstract}

\section{Definitions and Introduction}

In this paper, $M$ will be a hyperbolic Riemann surface with the unit disc as its universal cover, and $\Gamma$ is the covering group such that $M \simeq \mathbb{D} / \Gamma$. The Banach space $L^{1}(M)$ is the space of measurable functions on $M$ with norm $\|\varphi\|_{1}=\int_{M}|\varphi|<\infty$. Unless confusion arises, $\|\varphi\|$ will mean $\|\varphi\|_{1}$ in this paper. The Bergman space $A^{1}(M) \subset L^{1}(M)$ is the Banach space of holomorphic functions integrable on $M$. The Bers space $Q(M)$ is the Banach space of holomorphic quadratic differentials on $M$ with norm

$$
\|\varphi\|_{Q}=\sup _{z \in M} \rho_{M}^{-2}(z)|\varphi(z)|<\infty,
$$

where $\varphi \in Q$ and $\rho_{M}$ is the hyperbolic density on $M$. The space of absolutely summable sequences is

$$
l^{1}=\left\{\left(a_{0}, a_{1}, \ldots\right): a_{i} \in \mathbb{C}, \sum_{i=0}^{\infty}\left|a_{i}\right|<\infty\right\},
$$

and the space of bounded sequences is

$$
l^{\infty}=\left\{\left(a_{0}, a_{1}, \ldots\right): a_{i} \in \mathbb{C}, \sup _{i}\left|a_{i}\right|<\infty\right\} .
$$

The space $\left(l^{1}\right)_{n}$ is the $n$-dimensional subspace of $l^{1}$ with all terms, except possibly the first $n$, being 0 .

For Banach spaces $X_{1}, X_{2}, \ldots$ with norms $\left\|x_{i}\right\|_{i}$ (for $x_{i} \in X_{i}$ ) and $p>0$, it is possible to form the Banach space $\left(X_{1} \oplus X_{2} \oplus \ldots\right)_{p}$, with elements of the form

Received 7 October 2004; 1 September 2005.

2000 Mathematics Subject Classification 30F60. 
$\left(x_{1}, x_{2}, \ldots\right)$, for $x_{i} \in X_{i}$, and norm given by

$$
\left\|\left(x_{1}, x_{2}, \ldots\right)\right\|_{p}=\left(\sum_{i=1}^{\infty}\left\|x_{i}\right\|_{i}^{p}\right)^{1 / p} .
$$

In a Banach space $Z$, a subspace $X$ of $Z$ is said to be complemented if there exists another subspace $Y$ of $Z$ such that the direct sum decomposition $Z=X \oplus Y$ can be formed.

In $[6]$, Lindenstrauss and Pelczynski showed that for the unit disc $\mathbb{D}$, the Bergman space $A^{p}(\mathbb{D})$ is isomorphic to $l^{p}$ for $1 \leqslant p<\infty$ by using techniques from functional analysis and the fact that there is a bounded linear projection from $L^{p}(\mathbb{D})$ onto $A^{p}(\mathbb{D})$. In this paper, these techniques are adapted to extend their result to cover infinite-dimensional Bergman spaces of hyperbolic Riemann surfaces.

Coifman and Rochberg, in their paper [1], proved that there exists a sequence of points $\zeta_{1}, \zeta_{2}, \ldots$ in $\mathbb{D}$ such that if $f \in A^{1}(\mathbb{D})$, then there are complex numbers $\lambda_{1}, \lambda_{2}, \ldots$ that give a decomposition of $f$ by

$$
f(z)=\sum_{i=1}^{\infty} \lambda_{i} \varphi_{i}(z)
$$

where the $\varphi_{i}$ are given by

$$
\varphi_{i}(z)=\frac{\left(1-\left|\zeta_{i}\right|^{2}\right)^{2}}{\left(1-\overline{\zeta_{i}} z\right)^{4}}
$$

and $\sum_{i=1}^{\infty}\left|\lambda_{i}\right|<C_{1}\|f\|$, for some universal constant $C_{1}$. For a given $f$, the choice of $\lambda_{1}, \lambda_{2}, \ldots$ may not be unique. Conversely, if $\sum_{i=1}^{\infty}\left|\lambda_{i}\right|<\infty$, then $f$ given by the formula in $(1.1)$ is in $A^{1}(\mathbb{D})$, and $\|f\| \leqslant C_{2} \sum_{i=1}^{\infty}\left|\lambda_{i}\right|$ for some universal constant $C_{2}$. If the points $\zeta_{1}, \zeta_{2}, \ldots$ could be chosen so that each $f \in A^{1}(\mathbb{D})$ had a unique representation of the form (1.1), then there would be an explicit isomorphism between $A^{1}(\mathbb{D})$ and $l^{1}$ in terms of the coefficients of the expansion, and the corresponding $\varphi_{i}$ would be a basis for $A^{1}(\mathbb{D})$. It is an open question as to whether this can be done. Coifman and Rochberg actually proved their theorem for a wider class of domains than $\mathbb{D}$ and also for a wider range of $p$, that is, $0<p<\infty$ instead of just the $p=1$ case outlined above.

An explicit basis for $A^{1}(\mathbb{D})$ is given in a paper by Wojtaszczyk [10], where spline systems are used to construct unconditional bases for the classical Hardy spaces, $H^{p}(\mathbb{D})$, for $0<p \leqslant 1$. These systems also turn out to be bases for Bergman spaces $A^{p}(\mathbb{D})$ for $0<p \leqslant 1$, and characterising elements of the Bergman spaces as the coefficients of their expansion in terms of these bases gives an explicit isomorphism between $A^{p}(\mathbb{D})$ and $l^{p}$ for $0<p \leqslant 1$.

Moving onto Riemann surfaces, a Riemann surface $M$ is said to be of finite analytic type if it can be obtained from a compact Riemann surface of finite genus $g$ by deleting a finite number, $n$, of points. A Riemann surface of finite analytic type is of non-exceptional type if it is hyperbolic or, equivalently, if $2 g-2+n>0$. Using the Riemann-Roch theorem, it can be shown, see for example [3], that the dimension of the Bergman space $A^{1}(M)$ is finite if and only if $M$ is of finite analytic type. If $M$ is of non-exceptional finite analytic type, with genus $g$ and $n$ punctures, then the dimension of $A^{1}(M)$ is given by $3 g-3+n$.

This result says that the condition in the main theorem of this paper, that the dimension of $A^{1}(M)$ is infinite, only precludes those $M$ of finite analytic type. The main theorem is as follows. 
THEOREM (Theorem 2.6). If $M$ is a hyperbolic Riemann surface with $\operatorname{dim} A^{1}(M)=\infty$, then $A^{1}(M)$ is isomorphic to the sequence space $l^{1}$.

An outline of the proof is given below.

(1) For a suitable disjoint subdivision $M_{1}, M_{2}, \ldots$ of the Riemann surface $M$, define the surjective linear operator

$$
R: L^{1}(M) \rightarrow\left(L^{1}\left(M_{1}\right) \oplus L^{1}\left(M_{2}\right) \oplus \ldots\right)_{1},
$$

which acts by $R(f)=\left(R_{1}(f), R_{2}(f), \ldots\right)$ where $R_{i}$ is the restriction map given by $R_{i}(f)=\left.f\right|_{M_{i}}$.

(2) There exist projections $P_{i}$ of $L^{1}\left(M_{i}\right)$ onto itself that satisfy:

(i) $\left\|P_{i}\right\| \leqslant 1$;

(ii) $P_{i}\left(L^{1}\left(M_{i}\right)\right)$ is isometric to $\left(l^{1}\right)_{\alpha_{i}}$ for some integer $\alpha_{i}$;

(iii) $\left\|\left.\left(P_{i} \circ R_{i}-R_{i}\right)\right|_{A^{1}(M)}\right\| \leqslant \epsilon_{i}$ for a given $\epsilon_{i}>0$.

(3) The space $\Lambda=\left(P_{1}\left(L^{1}\left(M_{1}\right)\right) \oplus P_{2}\left(L^{1}\left(M_{2}\right)\right) \oplus \ldots\right)$ is isometric to $l^{1}$.

(4) The map $T: R\left(A^{1}(M)\right) \rightarrow \Lambda$ given by component-wise projecting with the $P_{i}$ satisfies $\|T-I\| \leqslant \sum_{i=1}^{\infty} \epsilon_{i}$ where $I$ is the identity on $R\left(A^{1}(M)\right)$.

(5) Functional analysis theory then shows that if the $\epsilon_{i}$ are made small enough, then $T\left(R\left(A^{1}(M)\right)\right)$ is complemented in $\Lambda$, and since $\Lambda$ is isometric to $l^{1}$, it follows that $A^{1}(M)$ is isomorphic to $l^{1}$. This relies on the theorem of Pelczynski, given in [5], which states that every infinite-dimensional complemented subspace of $l^{p}$ for $1 \leqslant p<\infty$ is isomorphic to $l^{p}$, and completes the proof.

If two spaces are isomorphic, then their respective Banach duals are also isomorphic. It is well known that the Banach dual of $l^{1}$ is the sequence space $l^{\infty}$, and it is also known (see, for example [7]) that the Banach dual of $A^{1}(M)$ is $Q(M)$. This characterisation of the Banach duals of the spaces in Theorem 2.6 gives the following corollary.

TheOREM (Theorem 2.7). If $M$ is a hyperbolic Riemann surface with $\operatorname{dim} A^{1}(M)=\infty$, then $Q(M)$ is isomorphic to the sequence space $l^{\infty}$.

This theorem has applications to Teichmüller theory which will be briefly outlined. For a fuller discussion and relevant definitions, refer to $\S 3$. However, the Teichmüller space $T(M)$ of a Riemann surface $M$ is biholomorphically equivalent, via the Bers embedding, to a subdomain of $Q(M)$. Therefore, Theorem 2.7 can be used to draw certain conclusions about $T(M)$ in the case where $M$ is of infinite analytic type.

The situation when $M$ is of non-exceptional finite analytic type is as follows. As stated earlier, the Riemann-Roch theorem gives the dimension of $A^{1}(M)$ as $3 g-3+n$. Since $Q(M)$ can be identified with the Banach dual of $A^{1}(M)$, then $Q(M)$ also has dimension $3 g-3+n$. In this case, $T(M)$ is biholomorphically equivalent to a subdomain of $\mathbb{C}^{3 g-3+n}$.

Before outlining the application of Theorem 2.7 to the case where $M$ is of infinite analytic type, recall that a Lipschitz mapping $f$ between two metric spaces $X_{1}, X_{2}$ with metrics $d_{1}, d_{2}$ satisfies the following condition for $x, y \in X_{1}$, and constants $C, \alpha$ independent of $x$ and $y$ :

$$
d_{2}(f(x), f(y)) \leqslant C d_{1}(x, y)^{\alpha} .
$$


A mapping $f$ is bi-Lipschitz if $f$ and its inverse are both Lipschitz, and is locally bi-Lipschitz if every $x \in X_{1}$ has a neighbourhood on which $f$ satisfies a Lipschitz condition.

The Bers embedding is actually a locally bi-Lipschitz map with respect to the Teichmüller metric on Teichmüller space, $T(M)$, and the metric arising from the Bers norm on the Bers space, $Q(M)$. Since an isomorphism is locally bi-Lipschitz, it follows that $Q(M)$ and $l^{\infty}$ are locally bi-Lipschitz equivalent spaces, which gives rise to the following theorem.

THEOREM (Theorem 3.2). If $M \simeq \mathbb{D} / \Gamma$ and $N \simeq \mathbb{D} / \Gamma_{1}$ are two hyperbolic Riemann surfaces with infinite-dimensional Bergman spaces, then their Teichmüller spaces are locally bi-Lipschitz equivalent.

Theorem 2.6 shows that if $M$ and $N$ are two Riemann surfaces with infinitedimensional Bergman spaces, then there exists an isomorphism between their Bergman spaces, since both $A^{1}(M)$ and $A^{1}(N)$ are isomorphic with $l^{1}$. Consider the following counterpoint to this result. A map between Bergman spaces $T: A^{1}(M) \rightarrow$ $A^{1}(N)$ is said to be geometric if there exists a conformal map $\alpha: M \rightarrow N$ and a complex number $\theta \in \mathbb{C}$ with $|\theta|=1$ such that for all $\varphi \in A^{1}(N)$,

$$
T^{-1}(\varphi)=\theta(\varphi \circ \alpha)\left(\alpha^{\prime}\right)^{2} \text {. }
$$

In [8], Markovic proved the following theorem.

Theorem A. Suppose that $M$ and $N$ are Riemann surfaces of non-exceptional type. Let $T: A^{1}(M) \rightarrow A^{1}(N)$ be a surjective linear isometry. Then the isometry $T$ is geometric. The surfaces $M$ and $N$ are conformally related and therefore homeomorphic.

From Theorem 2.6, if $A^{1}(M)$ and $A^{1}(N)$ are infinite dimensional, then they will be isomorphic. However, they cannot be isometric, according to Theorem A, unless $M$ and $N$ are conformally equivalent. Finally, the following conjecture is an interesting question on the global structure of Teichmüller space.

Conjecture B. If two Teichmüller spaces, of finite or infinite dimension, are globally bi-Lipschitz equivalent, then they are conformally equivalent.

\section{Isomorphism of infinite-dimensional Bergman spaces with sequence space $l^{1}$}

Sections 2.1-2.4 deal with the material needed for the proof of the main theorem of the section, Theorem 2.6. The theorem itself and its proof are given in $\S 2.5$.

\subsection{Bergman kernels}

The material in this section can be found in, for example, $[\mathbf{2}]$ or $[\mathbf{3}]$. The Bergman kernel on $\mathbb{D} \times \mathbb{D}$ is given by

$$
K(z, \zeta)=\frac{1}{(1-z \bar{\zeta})^{4}}
$$

and satisfies the following properties: 
(i) $K(z, \zeta)=\overline{K(\zeta, z)}$;

(ii) for every Möbius transformation $f: \mathbb{D} \rightarrow \mathbb{D}$,

$$
K(f(z), f(\zeta)) f^{\prime}(z)^{2} \overline{f^{\prime}(\zeta)^{2}}=K(z, \zeta) ;
$$

(iii) $\int_{\mathbb{D}}|K(z, \zeta)| d x d y \leqslant \pi \rho^{2}(\zeta)$;

(iv) for every $f \in A^{1}(\mathbb{D})$,

$$
f(z)=\frac{3}{\pi} \int_{\mathbb{D}} \rho^{-2}(\zeta) K(z, \zeta) f(\zeta) d \xi d \eta
$$

where $\rho(z)=2\left(1-|z|^{2}\right)^{-1}$ is the hyperbolic density on $\mathbb{D}$, and $\xi, \eta$ are coordinates in the $\zeta$-plane;

(v) for each $\zeta \in \mathbb{D}$,

$$
\sup _{z \in \mathbb{D}}|K(z, \zeta)| \rho^{-2}(z)<\infty .
$$

Proof of these facts. The first property is obvious from the definition of $K$. The second property follows from an elementary calculation. For the third property, consider

$$
g(\zeta)=\int_{\mathbb{D}}|K(z, \zeta)| d x d y
$$

and observe that under the change of variable $\zeta \mapsto f(\zeta)$, for a Möbius transformation $f: \mathbb{D} \rightarrow \mathbb{D}$, we have $g(f(\zeta))\left|f^{\prime}(\zeta)\right|^{2}=g(\zeta)$. Therefore, $g(\zeta)$ can be determined by evaluating $g(0)$. Since $g(0)=\pi$, this gives the third property with equality. For the fourth property, consider first the mean value property for harmonic functions, that is, for $r<1$,

$$
f(0)=\frac{1}{2 \pi} \int_{0}^{2 \pi} f\left(r e^{i \theta}\right) d \theta .
$$

Hence,

$$
f(0) \int_{0}^{1}\left(1-r^{2}\right) r d r=\frac{1}{2 \pi} \int_{0}^{2 \pi} \int_{0}^{1}\left(1-r^{2}\right) r f\left(r e^{i \theta}\right) d r d \theta,
$$

since $f$ is integrable in $\mathbb{D}$. This can now be rewritten as

$$
f(0)=\frac{3}{\pi} \int_{\mathbb{D}} \rho^{-2}(\zeta) K(0, \zeta) f(\zeta) d \xi d \eta,
$$

and the invariance properties of $\rho$ and $K$ under Möbius transformations give the general formula. The final property is obvious from the definitions of $K$ and $\rho$.

A group $\Gamma$ of self-homeomorphisms of $\mathbb{D}$ acts properly discontinuously on $\mathbb{D}$ if, for all compact sets $K \subseteq \mathbb{D}$, the set $\{A \in \Gamma: A(K) \cap K\}$ is finite. A group $\Gamma$ of holomorphic self-homeomorphisms of $\mathbb{D}$ that acts properly discontinuously on $\mathbb{D}$ is called a Fuchsian group.

Every hyperbolic Riemann surface $M$ has the disc $\mathbb{D}$ as its universal cover, that is, there is a Fuchsian covering group $\Gamma$ such that $M \simeq \mathbb{D} / \Gamma$. Now, given such a covering group $\Gamma$, form the Poincare theta series given by

$$
F(z, \zeta)=\sum_{\gamma \in \Gamma} K(\gamma(z), \zeta) \gamma^{\prime}(z)^{2} .
$$

The series for $F(z, \zeta)$ converges absolutely and uniformly on compact subsets of $\mathbb{D}$ to a function holomorphic in $z$, antiholomorphic in $\zeta$ and satisfies: 
(i) $F(z, \zeta)=\overline{F(\zeta, z)}$;

(ii) for $\gamma \in \Gamma, F(\gamma(z), \zeta) \gamma^{\prime}(z)^{2}=F(z, \zeta)$;

(iii) for $A$ in the normaliser of $\Gamma, F(A(z), A(\zeta)) A^{\prime}(z)^{2} \overline{A^{\prime}(\zeta)^{2}}=F(z, \zeta)$;

(iv) for every holomorphic quadratic differential $\psi \in A^{1}(\mathbb{D} / \Gamma)$ that respects the group $\Gamma$,

$$
\psi(z)=\frac{3}{\pi} \int_{\mathbb{D} / \Gamma} \rho^{-2}(\zeta) F(z, \zeta) \psi(\zeta) d \xi d \eta ;
$$

(v) for a fixed $|\zeta|<1$,

$$
\sup _{z \in \mathbb{D}}\left|F(z, \zeta) \rho^{-2}(z)\right|<\infty
$$

Remark. For the details of the proof, see [2]. The important fourth point, the integral reproducing formula, reduces to the case for the disc by the invariance of $F(z, \zeta)$ and $\rho(z)$ under the action of $\Gamma$.

For a hyperbolic Riemann surface $M \simeq \mathbb{D} / \Gamma$, there is a universal covering map $\pi: \mathbb{D} \rightarrow M$ such that $\pi \circ \gamma=\pi$, for all $\gamma \in \Gamma$. Pick a fundamental region $\Omega$ of $\mathbb{D} / M$ so that $\left.\pi\right|_{\Omega}$ is injective, and denote now $\left.\pi\right|_{\Omega}$ by $\pi$ without confusion. The hyperbolic density $\rho_{M}$ for the surface $M$ is defined by $\rho_{M}(\pi(z))\left|\pi^{\prime}(z)\right|=\rho(z)$.

Define the kernel function for $M$ by

$$
K_{M}(\pi(z), \pi(\zeta)) \pi^{\prime}(z)^{2} \overline{\pi^{\prime}(\zeta)^{2}}=F(z, \zeta) .
$$

Lemma 2.1. The kernel function $K_{M}: M \times M \rightarrow \mathbb{C}$ defined above is holomorphic in the first argument, antiholomorphic in the second argument and satisfies the following properties (here $\mu, \nu \in M)$ :

(i) $K_{M}(\mu, \nu)=\overline{K_{M}(\nu, \mu)}$;

(ii) for every conformal $f: M \rightarrow M, K_{M}(f(\mu), f(\nu)) f^{\prime}(\mu)^{2} \overline{f^{\prime}(\nu)^{2}}=K_{M}(\mu, \nu)$;

(iii) $\int_{M}\left|K_{M}(\mu, \nu)\right| d \mu \leqslant \pi \rho_{M}^{2}(\nu)$;

(iv) for every $\varphi \in A^{1}(M)$,

$$
\varphi(\mu)=\frac{3}{\pi} \int_{M} \rho_{M}^{-2}(\nu) K_{M}(\mu, \nu) \varphi(\nu) d \nu
$$

(v) for each fixed $\nu \in M$,

$$
\sup _{\mu \in M}\left|K_{M}(\mu, \nu)\right| \rho_{M}^{-2}(\mu)<\infty .
$$

Proof. Most of the properties follow from the analogous properties of $F$ (see [2]), and here we will just prove the third property since it will be used shortly:

$$
\begin{aligned}
\int_{M}\left|K_{M}(\pi(z), \nu)\right| d \nu & =\int_{\Omega}\left|K_{M}(\pi(z), \pi(\zeta))\right|\left|\pi^{\prime}(\zeta)^{2}\right| d \zeta \\
& =\int_{\Omega}|F(z, \zeta)|\left|\pi^{\prime}(z)^{-2}\right| d \zeta \leqslant\left|\pi^{\prime}(z)^{-2}\right| \int_{\mathbb{D}}|K(z, \zeta)| d \zeta \\
& \leqslant \pi\left|\pi^{\prime}(z)^{-2}\right| \rho(z)^{2}=\pi \rho_{M}(\pi(z))^{2},
\end{aligned}
$$

where $\nu=\pi(\zeta)$ for $\zeta \in \Omega$, a fundamental region for $M$ in $\mathbb{D}$. This completes the proof. 
Define the linear map $P: L^{1}(M) \rightarrow A^{1}(M)$ by

$$
(P(\varphi))(\mu)=\frac{3}{\pi} \int_{M} \rho_{M}^{-2}(\nu) K_{M}(\mu, \nu) \varphi(\nu) d \nu
$$

for $\mu, \nu \in M$. For any $\varphi \in L^{1}(M)$, it is clear that the integral formula for $P(\varphi)$ means that $P(\varphi)$ will be holomorphic, so the image of $P$ is indeed $A^{1}(M)$.

Theorem 2.2. There exists a bounded linear projection $\theta: L^{1}(M) \rightarrow A^{1}(M)$, given by $\theta: \varphi \mapsto P(\varphi)$ for $\varphi \in L^{1}(M)$.

Proof. The map $\theta$ is clearly linear, and bounded, since

$$
\begin{aligned}
\|P(\varphi)\| & =\int_{M}|P(\varphi(\mu))| d \mu=\frac{3}{\pi} \int_{M}\left|\int_{M} \rho_{M}^{-2}(\nu) K_{M}(\mu, \nu) \varphi(\nu) d \nu\right| d \mu \\
& \leqslant \int_{M}\left(\int_{M}\left|K_{M}(\mu, \nu)\right| d \mu\right) \rho_{M}^{-2}(\nu)|\varphi(\nu)| d \nu
\end{aligned}
$$

by Fubini's theorem, which we can apply by the fifth property in Lemma 2.1, and then using the third property of Lemma 2.1 gives

$$
\|P(\varphi)\| \leqslant 3 \int_{M}|\varphi(\nu)| d \nu
$$

Hence, $\|\theta\| \leqslant 3$. The integral reproducing formula given in $(2.1)$ shows that $\left.\theta\right|_{A^{1}(M)}$ is the identity, $\theta^{2}=\theta$, and so $\theta$ is a projection.

\subsection{Subdividing Riemann surfaces}

This section contains a recipe for subdividing a Riemann surface into a disjoint union of relatively compact subsets.

For every $p \in M$, there exists an open subset $U_{p} \subset M$ containing $p$, and a chart $\pi_{p}$ such that $\pi_{p}\left(U_{p}\right)$ is a disc in $\mathbb{C}$ and $\pi_{p}(p)=0$. Let $V_{p}$ be an open simply connected set in $M$ whose closure is contained in $U_{p}$, so that in particular $\pi_{p}\left(V_{p}\right)$ is a relatively compact subset of $\pi_{p}\left(U_{p}\right)$.

As $p$ varies through $M,\left(V_{p}\right)_{p \in M}$ forms an open cover of $M$, and it is possible to find a countable subset $p_{1}, p_{2}, \ldots$ such that

$$
M=\bigcup_{i=1}^{\infty} V_{p_{i}} .
$$

Now modify the subsets $V_{p_{i}}$ to give a disjoint partition of $M$ in the following way: define $M_{1}=V_{p_{1}}$, and then inductively,

$$
M_{n}=V_{p_{n}} \backslash\left(\bigcup_{i=1}^{n-1} V_{p_{i}}\right) .
$$

\subsection{Compactness of restriction operators}

Proposition 2.3. Let $K$ be a relatively compact subset of $\mathbb{D}$. Then the restriction operator $R_{K}: A^{1}(\mathbb{D}) \rightarrow A^{1}(K)$, given by $R(f)=\left.f\right|_{K}$ for $f \in A^{1}(\mathbb{D})$, is compact.

Proof. The restriction operator $R_{K}$ is compact if and only if for every bounded sequence $f_{n} \in A^{1}(\mathbb{D})$, the sequence $R_{K}\left(f_{n}\right)$ has a convergent subsequence. 
For $z \in \mathbb{D}$, let $d(z, \partial \mathbb{D})$ be the shortest Euclidean distance from $z$ to the boundary of $\mathbb{D}$, and similarly let $d(K, \partial \mathbb{D})=\inf _{z \in K} d(z, \partial \mathbb{D})$. The Cauchy integral formula gives

$$
\left|f\left(z_{0}\right)\right| \leqslant \frac{1}{\pi t^{2}} \int_{0}^{2 \pi} \int_{0}^{t}\left|f\left(z_{0}+r e^{i \theta}\right)\right| r d r d \theta
$$

for $t<d\left(z_{0}, \partial \mathbb{D}\right)$ and $f \in A^{1}(\mathbb{D})$. Therefore,

$$
\left|f\left(z_{0}\right)\right|\left(\pi t^{2}\right) \leqslant \int_{\mathbb{D}}|f|,
$$

and this holds for $t<d\left(z_{0}, \partial \mathbb{D}\right)$, so in particular, for any $z_{0} \in K$,

$$
\left|f\left(z_{0}\right)\right| \leqslant \frac{1}{\pi(d(K, \partial \mathbb{D}))^{2}} \int_{\mathbb{D}}|f| .
$$

Now, let $f_{n}$ be a bounded sequence in $A^{1}(\mathbb{D})$ and without loss of generality, $\left\|f_{n}\right\| \leqslant 1$ for all $n$. We can find a relatively compact subset $\Omega \subset \mathbb{D}$ such that $K \subset \Omega$. Since $\left\|f_{n}\right\| \leqslant 1,(2.2)$ implies that $\left|f_{n}(z)\right| \leqslant C_{\Omega}$ for all $n$, for all $z \in \Omega$, and where

$$
C_{\Omega}=\left(\pi(d(K, \partial \mathbb{D}))^{2}\right)^{-1} .
$$

This shows that $R_{\Omega}\left(f_{n}\right)$ is uniformly bounded on $\Omega$, which means that $R_{\Omega}\left(f_{n}\right)$ is a normal family (see, for example, [9]). Hence, there is a subsequence $R_{\Omega}\left(f_{n_{k}}\right)$ that converges uniformly on compact subsets of $\Omega$ and, in particular, uniformly on $K$. Uniform convergence implies convergence in the $L^{1}$ norm, so there exists some function $g \in A^{1}(K)$ such that $R_{K}\left(f_{n_{k}}\right) \rightarrow g$.

Recall that given a Riemann surface $M$, we have a disjoint partition from $\S 2.2$ of $M=\bigsqcup_{i=1}^{\infty} M_{i}$.

Corollary 2.4. Let $R_{i}: A^{1}(M) \rightarrow A^{1}\left(M_{i}\right)$ be the restriction operator given by $R_{i}(f)=\left.f\right|_{M_{i}}$ for $f \in A^{1}(M)$. Then $R_{i}$ is a compact operator.

Proof. With the notation of $\S 2.2, M_{i} \subset U_{p_{i}}$, and $\pi_{p_{i}}\left(M_{i}\right)$ is a relatively compact subset of the $\operatorname{disc} \pi_{p_{i}}\left(U_{p_{i}}\right) \subset \mathbb{C}$. The function $\widetilde{f}=f \circ\left(\pi_{p_{i}}\right)^{-1}$ defined on $\pi_{p_{i}}\left(U_{p_{i}}\right)$ is analytic, so it is possible to lift functions in $A^{1}\left(U_{p_{i}}\right)$ to functions in $A^{1}\left(\pi_{p_{i}}\left(U_{p_{i}}\right)\right)$. By the previous proposition, the restriction operator given by $R_{\pi_{p_{i}}\left(M_{i}\right)}: A^{1}\left(\pi_{p_{i}}\left(U_{p_{i}}\right)\right) \rightarrow A^{1}\left(\pi_{p_{i}}\left(M_{i}\right)\right)$ is compact, and so $R_{i}$ must also be compact.

\subsection{Projections on $L^{1}$}

In this section, we consider $\Omega$ to be a simply connected, relatively compact subset of a Riemann surface $M$, but, via the Riemann map, we can for simplicity assume that $\Omega$ is a bounded simply connected plane domain. Subdivide $\Omega$ into a finite number of subsets, $\Omega_{1}, \ldots, \Omega_{n}$. For a given $f \in L^{1}(\Omega)$, define $\lambda_{i}$ to be $\int_{\Omega_{i}} f$. We have

$$
\sum_{i=1}^{n}\left|\lambda_{i}\right|=\sum_{i=1}^{n}\left|\int_{\Omega_{i}} f\right| \leqslant \int_{\Omega}|f|<\infty .
$$


Define the map $P: L^{1}(\Omega) \rightarrow L^{1}(\Omega)$ by

$$
P(f)=\sum_{i=1}^{n} \frac{\lambda_{i}}{m\left(\Omega_{i}\right)} \mathbf{1}_{\Omega_{i}}
$$

where $\mathbf{1}_{\Omega_{i}}$ denotes the indicator function of $\Omega_{i}$, and $m$ is the usual two-dimensional Lebesgue measure of $\Omega_{i}$. The map $P$ is clearly linear and bounded $(\|P\| \leqslant 1$ in fact), and also a projection, since $P^{2}=P$.

We can define a map $\mu: P\left(L^{1}(\Omega)\right) \rightarrow\left(l^{1}\right)_{n}$ given by

$$
\mu(P(f))=\left(\lambda_{1}, \ldots, \lambda_{n}, 0, \ldots\right) .
$$

Now, $\|\mu(P(f))\|_{l^{1}}=\sum_{i=1}^{n}\left|\lambda_{i}\right|$. Also,

$$
\|P(f)\|_{1}=\int_{\Omega}|P(f)|=\int_{\Omega}\left|\sum_{i=1}^{n} \frac{\lambda_{i}}{m\left(\Omega_{i}\right)} \mathbf{1}_{\Omega_{i}}\right|=\sum_{i=1}^{n} \int_{\Omega_{i}}\left|\frac{\lambda_{i}}{m\left(\Omega_{i}\right)}\right|=\sum_{i=1}^{n}\left|\lambda_{i}\right|
$$

since the supports of $\mathbf{1}_{\Omega_{i}}$ are disjoint. Hence, $\mu$ is isometric, and so $P\left(L^{1}(\Omega)\right)$ is isometric to $\left(l^{1}\right)_{n}$.

We now give a discussion to show that we can find a fine enough subdivision of $\Omega$ so that for the corresponding projection $P,\|P(f)-f\|<\epsilon$ for $f \in A^{1}(\Omega)$ with $\|f\| \leqslant 1$. Since $\Omega$ is relatively compact in $M, \sup \{|f(z)|\}$ is bounded, where the supremum is taken over all $f \in A^{1}(M)$ with $\|f\| \leqslant 1$ and over all $z \in \Omega$ (recall the proof of Proposition 2.3). This means that

$$
\Theta=\left\{\left.f\right|_{\Omega}: f \in A^{1}(M),\|f\| \leqslant 1\right\}
$$

is a normal family, and hence is equicontinuous; that is, for all $f \in \Theta$ and for all $\epsilon>0$, there exists a $\delta>0$ such that if $\left|z-z_{0}\right|<\delta$, for $z, z_{0} \in \Omega$, then $\left|f(z)-f\left(z_{0}\right)\right|<\epsilon$.

If $B\left(z_{i}, \delta\right)$ is a ball centred at $z_{i}$ of Euclidean radius $\delta$, then for any holomorphic function $f$,

$$
\int_{B\left(z_{i}, \delta\right)} f=f\left(z_{i}\right)
$$

If, now, $\Omega$ is subdivided into $\Omega_{1}, \ldots, \Omega_{n}$, with each $\Omega_{i} \subset B\left(z_{i}, \delta\right)$ for some $z_{i}$, and $P$ is the corresponding projection to this subdivision, then

$$
\int_{\Omega_{i}}|f-P(f)| \leqslant \int_{B\left(z_{i}, \delta\right)}\left|f(z)-f\left(z_{i}\right)\right|<\epsilon m\left(B\left(z_{0}, \delta\right)\right)
$$

recalling that $m\left(B\left(z_{i}, \delta\right)\right)$ is the area of $B\left(z_{i}, \delta\right)$, and noting that the last inequality follows from the equicontinuity of $\Theta$. Hence,

$$
\int_{\Omega}|f-P(f)|<\epsilon m(\Omega)
$$

and since we are assuming that $m(\Omega)$ is finite, and $\epsilon$ can be made as small as required, then we have the desired conclusion that $\|P-I\|$ can be as small as desired for $P$ corresponding to a suitably fine subdivision of $\Omega$.

The following proposition will be needed for the proof of Theorem 2.6.

Proposition 2.5. Let $S$ be a projection on a Banach space $X$. There exists an $\epsilon>0$ small enough so that if $T$ is another projection on $X$ satisfying

$$
\left\|\left.(T-I)\right|_{\operatorname{Im}(S)}\right\|<\epsilon
$$


where $I$ is the identity operator, and $\operatorname{Im}(S)$ denotes the image of $S$, then there is a projection from $X$ onto $\operatorname{Im}(T \circ S)$.

Proof. Let $f \in \operatorname{Im}(T \circ S)$, then by definition $f=T(g)$ for some $g \in \operatorname{Im}(S)$. Equation (2.3) implies that if we consider the operator $T$ restricted to have domain $\operatorname{Im}(S)$ and range $\operatorname{Im}(T \circ S)$, then we can find a left inverse $\widetilde{T}: \operatorname{Im}(T \circ S) \rightarrow \operatorname{Im}(S)$ for $T$, that is, if $h \in \operatorname{Im}(S)$, then

$$
\widetilde{T} \circ T(h)=h .
$$

In particular, $\widetilde{T}(f)=\widetilde{T}(T(g))=g$. Since $T$ is a projection, $\|T\| \leqslant 1$, which implies that $\widetilde{T}$ is bounded, and $\|g\| \leqslant\|\widetilde{T}\|\|f\|$. By the triangle inequality, and recalling $f \in \operatorname{Im}(T \circ S)$,

$$
\|S(f)-f\| \leqslant\|S(f)-g\|+\|g-f\| .
$$

However, since $f=T(g)$, we can use (2.3) to write the second term on the righthand side of (2.4) as

$$
\|g-f\|=\|g-T(g)\|<\epsilon\|g\| \leqslant \epsilon\|\widetilde{T}\|\|f\| .
$$

Since $g \in \operatorname{Im}(S)$, and since $S$ is a projection, we have $S(g)=g$. Thus, the first term on the right-hand side of (2.4) becomes

$$
\|S(f)-g\|=\|S(f)-S(g)\| \leqslant\|S\|\|f-g\| \leqslant \epsilon\|S\|\|\widetilde{T}\|\|f\|,
$$

by using (2.5). Therefore,

$$
\|S(f)-f\| \leqslant \epsilon(1+\|S\|)\|\widetilde{T}\|\|f\| .
$$

Since $S$ is a projection, it is bounded, and we know from above that $\widetilde{T}$ is bounded. Therefore, for $\epsilon$ small enough, we can find a left inverse for $S$ on $\operatorname{Im}(T \circ S)$. Since $S$ restricted to $\operatorname{Im}(T \circ S)$ is invertible, and $T$ restricted to $\operatorname{Im}(S)$ is invertible, it follows that $\operatorname{Im}(S \circ T \circ S)=\operatorname{Im}(S)$. Therefore, there exists an operator $\widetilde{S}$ : $\operatorname{Im}(S) \rightarrow \operatorname{Im}(T \circ S)$ such that for any $h \in \operatorname{Im}(T \circ S)$,

$$
\widetilde{S} \circ S(h)=h .
$$

In conclusion, $\widetilde{S} \circ S$ is a projection from $X$ onto $\operatorname{Im}(T \circ S)$, since it is bounded, linear, an idempotent and has image $\operatorname{Im}(T \circ S)$.

\subsection{Theorem 2.6 and its proof}

We are now ready to state our main theorem.

Theorem 2.6. If $M$ is a hyperbolic Riemann surface with $\operatorname{dim} A^{1}(M)=\infty$, then $A^{1}(M)$ is isomorphic to the sequence space $l^{1}$.

Proof. Given a surface $M$, subdivide $M$ into relatively compact subsets $M_{i}$ as described in $\S 2.2$, so that $M=\bigsqcup_{i=1}^{\infty} M_{i}$. Let $R_{i}: L^{1}(M) \rightarrow L^{1}\left(M_{i}\right)$ be the restriction map given by $R_{i}(f)=\left.f\right|_{M_{i}}$, for $f \in L^{1}(M)$.

Define the operator $R: L^{1}(M) \rightarrow\left(L^{1}\left(M_{1}\right) \oplus L^{1}\left(M_{2}\right) \oplus \ldots\right)_{1}$ by

$$
R(f)=\left(R_{1}(f), R_{2}(f), \ldots\right)
$$


for $f \in L^{1}(M)$. The operator $R$ is isometric, since

$$
\|R(f)\|:=\sum_{i=1}^{\infty}\left\|R_{i}(f)\right\|=\sum_{i=1}^{\infty} \int_{M_{i}}|f|=\int_{M}|f|=\|f\|,
$$

and $R$ is also clearly surjective. By the considerations in $\S 2.3,\left.R_{i}\right|_{A^{1}(M)}$ is a compact operator. Let $\Delta=\left\{f \in A^{1}(M):\|f\| \leqslant 1\right\}$. Then $R_{i}(\Delta)$ is a totally bounded set in $L^{1}\left(M_{i}\right)$ by the compactness of $\left.R_{i}\right|_{A^{1}(M)}$. Now, given $\epsilon_{i}>0$, by considerations in $\S 2.4$, we can find a projection $P_{i}$ of $L^{1}\left(M_{i}\right)$ into itself satisfying $\left\|P_{i}\right\| \leqslant 1$, $P_{i}\left(L^{1}\left(M_{i}\right)\right)$ is isometric to $\left(l^{1}\right)_{\alpha_{i}}$ for some $\alpha_{i} \in \mathbb{Z}^{+}$, and $\left\|P_{i}\left(R_{i}(f)\right)-R_{i}(f)\right\| \leqslant \epsilon_{i}$ for all $f \in \Delta$.

Let $\Lambda=\left(P_{1}\left(L^{1}\left(M_{1}\right)\right) \oplus P_{2}\left(L^{1}\left(M_{2}\right)\right) \oplus \ldots\right)_{1}$, a subspace of $\left(L^{1}\left(M_{1}\right) \oplus L^{1}\left(M_{2}\right) \oplus\right.$ ... $)_{1}$. Since each $P_{i}\left(L^{1}\left(M_{i}\right)\right)$ is isometric to $\left(l^{1}\right)_{\alpha_{i}}$ for some $\alpha_{i} \in \mathbb{Z}^{+}, \Lambda$ is isometric to $l^{1}$. Define the operator $T: R\left(A^{1}(M)\right) \rightarrow \Lambda$ by

$$
T\left(R_{1}(f), R_{2}(f), \ldots\right)=\left(P_{1}\left(R_{1}(f)\right), P_{2}\left(R_{2}(f)\right), \ldots\right) .
$$

Since the dimension of $A^{1}(M)$ is infinite, $R\left(A^{1}(M)\right)$ also must be infinite dimensional. We also have

$$
\|T(\xi)-\xi\| \leqslant\left(\sum_{i=1}^{\infty} \epsilon_{i}\right)\|\xi\|
$$

for $\xi \in R\left(A^{1}(M)\right)$, and so given $\epsilon>0$, it is possible to choose the $\left(\epsilon_{i}\right)_{i}$ so that $\|T(\xi)-\xi\|<\epsilon\|\xi\|$, for $\xi \in R\left(A^{1}(M)\right)$.

By Theorem 2.2, there exists a bounded linear projection $\theta: L^{1}(M) \rightarrow A^{1}(M)$. Therefore, there is a bounded linear projection $\widetilde{\theta}: R\left(L^{1}(M)\right) \rightarrow R\left(A^{1}(M)\right)$, given by

$$
\widetilde{\theta}\left(R_{1}(f), R_{2}(f), \ldots\right)=\left(R_{1}(\theta(f)), R_{2}(\theta(f)), \ldots\right)
$$

which is clearly linear, bounded and satisfies $\widetilde{\theta}^{2}=\widetilde{\theta}$. Therefore, $R\left(A^{1}(M)\right)$ is complemented in $R\left(L^{1}(M)\right)$. Thus, by Proposition 2.5, if $\epsilon$ is small enough, $T\left(R\left(A^{1}(M)\right)\right)$ is complemented in $R\left(L^{1}(M)\right)$ and, in particular, $\Lambda$.

If $\epsilon<1$, then $\|T-I\|<1$, and by a standard result, $T$ is thus invertible and an isomorphism. Every infinite-dimensional complemented subspace of $l^{1}$ is isomorphic to $l^{1}$ (see $[5]$ ), and so $A^{1}(M)$ is isomorphic to $l^{1}$.

REMARK. Since this paper concentrates on the applications of Theorem 2.6 to Teichmüller theory in $\S 3$, only the result that $A^{1}(M)$ is isomorphic to $l^{1}$, for $M$ of infinite analytic type, is presented. However, the technique used in the proof can be adapted to show that, for such a Riemann surface $M, A^{p}(M)$ is isomorphic to $l^{p}$ for $1 \leqslant p<\infty$.

Let $\alpha_{\Gamma}: A^{1}(M) \rightarrow l^{1}$ be the isomorphism in Theorem 2.6. This induces an isomorphism of the Banach duals $\alpha_{\Gamma}^{*}:\left(l^{1}\right)^{*} \rightarrow\left(A^{1}(M)\right)^{*}$. It is well known that the Banach dual of $l^{1}$ can be identified with $l^{\infty}$. Furthermore, let $\Omega$ be a plane domain whose boundary consists of at least three distinct finite points. Then to each bounded linear functional $\Phi$ on $A^{1}(\Omega)$, there corresponds a unique $g \in Q(\Omega)$ such that

$$
\Phi(f)=\int_{\Omega} \rho_{\Omega}^{-2}(w) f(w) \overline{g(w)} d u d v
$$


for all $f \in A^{1}(\Omega)$. Moreover, $\frac{1}{3}\|g\|_{Q} \leqslant\|\Phi\| \leqslant\|g\|_{Q}$. Thus, if $M$ is a hyperbolic Riemann surface, then the Banach dual of $A^{1}(M)$ can be identified with $Q(M)$. A proof of this result can be found in, for example, [7]. This immediately gives us the following results.

TheOREM 2.7. If $M$ is a hyperbolic Riemann surface with $\operatorname{dim} A^{1}(M)=\infty$, then $Q(M)$ is isomorphic to the sequence space $l^{\infty}$.

Corollary 2.8. If $M$ and $N$ are two hyperbolic Riemann surfaces with infinite-dimensional Berman spaces, then $A^{1}(M)$ and $A^{1}(N)$ are isomorphic, and $Q(M)$ and $Q(N)$ are isomorphic.

\section{Application to Teichmüller theory}

We first give a brief introduction to Teichmüller theory in $\S 3.1$, and in particular the Bers embedding of Teichmüller space in $\S 3.2$. This material can be found in greater detail in, for example, $[\mathbf{3}]$ or $[\mathbf{4}]$. Section 3.3 gives the proof that infinitedimensional Teichmüller spaces are locally bi-Lipschitz equivalent.

\subsection{Teichmüller spaces}

If $\Omega \subset \mathbb{C}$ is a plane domain, a homeomorphism $f: \Omega \rightarrow f(\Omega)$ is quasiconformal if there exists $k<1$ such that $f$ has locally integrable distributional derivatives $f_{z}, f_{\bar{z}}$ on $\Omega$ and $\left|f_{\bar{z}}\right| \leqslant k\left|f_{z}\right|$ almost everywhere on $\Omega$. The map $f$ is then $K$-quasiconformal, where $K=(1+k) / 1-k$. If $k$ is the smallest such that the condition above is satisfied, then $K(f)=(1+k) /(1-k)$.

The complex dilatation of $f$ is $\mu(z)=f_{\bar{z}}(z) / f_{z}(z)$. For a general $\mu \in L^{\infty}(\Omega)$ satisfying $\|\mu\|_{\infty}<1$, the Beltrami equation is $f_{\bar{z}}(z)=\mu(z) f_{z}(z)$. The Beltrami equation can be solved by a quasiconformal map, which is unique up to post-composition by a Möbius transformation. Hence, there is a one-to-one correspondence between Möbius equivalent classes of quasiconformal homeomorphisms of $\Omega$ and the open unit ball $B(\Omega)$ of $L^{\infty}(\Omega)$.

Quasiconformality is a well-defined notion for Riemann surfaces and, in this case, Beltrami differentials are $(-1,1)$ differential forms on Riemann surfaces. If $f_{0}$ : $M \rightarrow N_{0}$ and $f_{1}: M \rightarrow N_{1}$ are quasiconformal maps from the Riemann surface $M$ to the Riemann surfaces $N_{0}$ and $N_{1}$, then $f_{0}$ is Teichmüller equivalent to $f_{1}$ if there exists a conformal map $g: N_{0} \rightarrow N_{1}$, and a homotopy through quasiconformal self-maps $h_{t}$, for $0 \leqslant t \leqslant 1$, of $M$ such that $h_{0}$ is the identity, $h_{1}=f_{1}^{-1} \circ g \circ f_{0}$ and $h_{t}(p)=p$ for all $0 \leqslant t \leqslant 1$ and for all $p \in \partial M$. Teichmüller space $T(M)$ is the space of equivalence classes of all quasiconformal maps on $M$ under the Teichmüller equivalence relation.

The definition of Teichmüller space can also be formulated in terms of Beltrami differentials in $B(M)$, the open unit ball of $L^{\infty}(M)$. In that case, two Beltrami differentials are Teichmüller equivalent if the corresponding quasiconformal mappings obtained from solving the Beltrami equation are Teichmüller equivalent. Denote by $[f]$ (respectively $[\mu]$ ) the Teichmüller class of a quasiconformal map $f$ (or Beltrami differential $\mu$ ).

The Teichmüller distance on $T(M)$ is given by

$$
d([f],[g])=\frac{1}{2} \inf \log K\left(\widetilde{f} \circ(\widetilde{g})^{-1}\right)
$$


where the infimum is taken over all quasiconformal maps in the equivalence classes of $f$ and $g$. It turns out that the Teichmüller metric is the same as the Kobayashi metric, which is defined as the largest (pseudo-)metric on $T(M)$ such that holomorphic mappings are non-increasing.

\subsection{The Bers embedding}

Let $B(\Gamma)=\left\{\mu \in L^{\infty}(\mathbb{D}): \mu(z)=\mu(\gamma(z)) \overline{\gamma^{\prime}(z)} / \gamma^{\prime}(z), \gamma \in \Gamma,\|\mu\|_{\infty}<1\right\}$ be the open unit ball of Beltrami differentials which respect the group $\Gamma$. If $M \simeq \mathbb{D} / \Gamma$, then $\mu \in B(\Gamma)$ if and only if there is a $\widehat{\mu} \in B(M)$ such that $\widehat{\mu}(\pi(z))=\mu(z) \overline{\pi^{\prime}(z)} / \pi^{\prime}(z)$. In this section $B(\Gamma), T(\Gamma)$, and so on, will be used.

Denote by $w_{\mu}$ the unique quasiconformal self-map of $\mathbb{D}$ with Beltrami coefficient $\mu$ and fixing $1,-1, i$. By standard theory, $w_{\mu}$ extends continuously to $\partial \mathbb{D}$. Denote by $w^{\mu}$ the quasiconformal self-map of $\overline{\mathbb{C}}$ with Beltrami coefficient $\mu$ in $\mathbb{D}$ and 0 in $\overline{\mathbb{C}} \backslash \mathbb{D}$, and which fixes $1,-1, i$. Restricted to $\mathbb{C} \backslash \mathbb{D}, w^{\mu}$ is conformal. It is true that $w_{\mu}=w_{\nu}$ if and only if $w^{\mu}=w^{\nu}$.

The Schwarzian derivative of an analytic function $f$ is defined by

$$
S(f)(z)=\left(\frac{f^{\prime \prime}}{f^{\prime}}\right)^{\prime}-\frac{1}{2}\left(\frac{f^{\prime \prime}}{f^{\prime}}\right)^{2} .
$$

If $\mu \in B(\Gamma)$, then $S\left(w^{\mu}\right)(z)=\varphi$ is a holomorphic quadratic differential for $\Gamma$ on $\overline{\mathbb{C}} \backslash \mathbb{D}$.

Recall that the Bers space $Q(\Gamma)$ on a plane domain $\Omega$, conformally equivalent to $\mathbb{D}$, is the space of holomorphic functions $\varphi$ in $\Omega$ such that $\varphi(\gamma(z)) \gamma^{\prime}(z)^{2}=$ $\varphi(z)$ for all $z \in \Omega$ and $\gamma \in \Gamma$, and also with finite Bers norm, that is, $\|\varphi\|_{Q}=$ $\sup _{z \in \Omega}|\varphi| \rho_{\Omega}^{-2}(z)<\infty$, where $\rho_{\Omega}$ is the hyperbolic density on $\Omega$.

Let $\Phi$ be the map on $B(\Gamma)$ defined by $\Phi(\mu)=S\left(w^{\mu}\right)$. The map $\Phi$ induces a one-to-one map $\widetilde{\Phi}: T(\Gamma) \rightarrow Q(\Gamma)$, which is called the Bers embedding. The Bers embedding maps $T(\Gamma)$ onto an open set in $Q(\Gamma)$, which is contained in the ball of radius $\frac{3}{2}$ in the Bers norm. Also, the image contains the ball of radius $\frac{1}{2}$.

If $U_{\Gamma}=\left\{[\mu] \in T(\Gamma): d([0],[\mu])<\frac{1}{2} \log 2\right\}$, where [0] is the Teichmüller class of the identity map, then $\left.\widetilde{\Phi}\right|_{U_{\Gamma}}$ is a homeomorphism onto an open set contained in the ball of radius $\frac{1}{2}$ and containing the ball of radius $\frac{1}{6}$ in the Bers norm. So $\widetilde{\Phi}$ gives a local coordinate near the origin of Teichmüller space.

It is possible to find charts for a neighbourhood of any point of Teichmüller space in the following way. Let $[\mu] \in T(\Gamma)$, and let $\Gamma_{1}=w_{\mu} \circ \Gamma \circ\left(w_{\mu}\right)^{-1}$ (recall that $w_{\mu}$ is the quasiconformal self-map of $\mathbb{D}$ fixing $1,-1, i)$. The Bers spaces $Q(\Gamma)$ and $Q\left(\Gamma_{1}\right)$ are isomorphic.

Let $\widetilde{\Phi_{1}}$ be the chart for $T\left(\Gamma_{1}\right)$ in $Q\left(\Gamma_{1}\right)$ described above. The maps $\widetilde{\Phi_{1}}$ and $\widetilde{\Phi}$ both give local coordinates, if they are restricted to neighbourhoods of Teichmüller distance less than $\frac{1}{2} \log 2$ from the origin in their respective spaces, $T(\Gamma)$ and $T\left(\Gamma_{1}\right)$. The map $F:[\nu] \mapsto[\sigma]$, where $w_{\sigma}=w_{\nu} \circ w_{\mu}$, is an isometric isomorphism from $T\left(\Gamma_{1}\right)$ to $T(\Gamma)$, which maps the equivalence class of the identity in $T\left(\Gamma_{1}\right)$ to the equivalence class of $w_{\mu}$ in $T(\Gamma)$. Thus, a chart for the neighbourhood of the origin in $T\left(\Gamma_{1}\right)$ is a chart for the neighbourhood of $[\mu]$ in $T(\Gamma)$. Where these charts overlap, the corresponding transition maps are holomorphic, which implies that Teichmüller space $T(\Gamma)$ is a complex Banach manifold modelled on $Q(\Gamma)$.

With respect to this structure on $T(\Gamma)$, the Bers embedding $\widetilde{\Phi}: T(\Gamma) \rightarrow Q(\Gamma)$ is a biholomorphic mapping. In particular, with respect to the Teichmüller metric 
on $T(\Gamma)$ and the metric arising from the Bers norm on $Q(\Gamma)$, the Bers embedding is a locally bi-Lipschitz mapping (recall (1.2) for the definition of a bi-Lipschitz mapping).

\subsection{Locally bi-Lipschitz equivalent Teichmüller spaces}

We have the following situation,

$$
\widetilde{\Phi}: T(\Gamma) \hookrightarrow Q(\Gamma), \quad \alpha_{\Gamma}^{*}: Q(\Gamma) \rightarrow l^{\infty}
$$

where the image of $\widetilde{\Phi}$ is contained in $Q(\Gamma)$. Since $\widetilde{\Phi}$ is a locally bi-Lipschitz mapping, there exists a neighbourhood, $X_{\Gamma}$, of the identity class in $T(\Gamma)$ such that $\left.\widetilde{\Phi}\right|_{X_{\Gamma}}$ is bi-Lipschitz. Since $\alpha_{\Gamma}^{*}$ is an isomorphism, $X_{\Gamma}$ is mapped onto a neighbourhood of the origin of $l^{\infty}$ by $\alpha_{\Gamma}^{*} \circ \widetilde{\Phi}$. If $Y_{\Gamma}=\left(\alpha_{\Gamma}^{*} \circ \widetilde{\Phi}\right)\left(X_{\Gamma}\right)$, then $X_{\Gamma}$ and $Y_{\Gamma}$ are bi-Lipschitz equivalent.

Note that in any statement where the term 'bi-Lipschitz' is used, this also implies that 'homeomorphic' holds for that statement. For example, $X_{\Gamma}$ and $Y_{\Gamma}$ are homeomorphic.

Lemma 3.1. If $M \simeq \mathbb{D} / \Gamma$ and $N \simeq \mathbb{D} / \Gamma_{1}$ are two hyperbolic Riemann surfaces with infinite-dimensional Bergman spaces, then a neighbourhood of the identity class in $T(\Gamma)$ is bi-Lipschitz equivalent to a neighbourhood of the identity class in $T\left(\Gamma_{1}\right)$.

Proof. Consider the neighbourhoods of the identity class in the respective Teichmüller spaces given by $X_{\Gamma}$ and $X_{\Gamma_{1}}$, and consider their images in $l^{\infty}$ under the respective maps $\alpha_{\Gamma}^{*} \circ \widetilde{\Phi}$ and $\alpha_{\Gamma_{1}}^{*} \circ \widetilde{\Phi_{1}}$, given by $Y_{\Gamma}$ and $Y_{\Gamma_{1}}$.

$$
T(\Gamma) \stackrel{\widetilde{\Phi}}{\longrightarrow} Q(\Gamma) \stackrel{\alpha_{\Gamma}^{*}}{\longrightarrow} l^{\infty} \stackrel{\alpha_{\Gamma_{1}}^{*}}{\longleftarrow} Q\left(\Gamma_{1}\right) \stackrel{\widetilde{\Phi_{1}}}{\longleftarrow} T\left(\Gamma_{1}\right) .
$$

The sets $Y_{\Gamma}$ and $Y_{\Gamma_{1}}$ are both open neighbourhoods of the origin in $l^{\infty}$, and so $Y:=Y_{\Gamma} \cap Y_{\Gamma_{1}}$ is also an open neighbourhood of the origin. Since $\alpha_{\Gamma}^{*} \circ \widetilde{\Phi}$ is a biLipschitz mapping of $X_{\Gamma}$, it has an inverse on $Y$, and $\left(\left(\alpha_{\Gamma}^{*} \circ \widetilde{\Phi}\right)^{-1}\right)(Y) \subseteq X_{\Gamma}$ is an open neighbourhood of the origin in $T(\Gamma)$.

Thus, $\left(\alpha_{\Gamma_{1}}^{*} \circ \widetilde{\Phi_{1}}\right) \circ\left(\alpha_{\Gamma}^{*} \circ \widetilde{\Phi}\right)^{-1}$ is a bi-Lipschitz mapping from a neighbourhood of the identity class in $T(\Gamma)$, namely $\left(\left(\alpha_{\Gamma}^{*} \circ \widetilde{\Phi}\right)^{-1}\right)(Y)$, to a neighbourhood of the identity class in $T\left(\Gamma_{1}\right)$, namely $\left(\alpha_{\Gamma_{1}}^{*} \circ \widetilde{\Phi_{1}}\right)(Y)$.

Theorem 3.2. If $M \simeq \mathbb{D} / \Gamma$ and $N \simeq \mathbb{D} / \Gamma_{1}$ are two hyperbolic Riemann surfaces with infinite-dimensional Bergman spaces, then their Teichmüller spaces are locally bi-Lipschitz equivalent.

Proof. Recall the discussion in $\S 3.2$, which says that a chart for the neighbourhood of the identity class in $T\left(w_{\mu} \circ \Gamma \circ\left(w_{\mu}\right)^{-1}\right)$ is a chart for the neighbourhood of $[\mu]$ in $T(\Gamma)$. Thus, charts for any $[\mu] \in T(\Gamma)$ and $[\nu] \in T\left(\Gamma_{1}\right)$ correspond to charts for the respective identity classes in $T\left(w_{\mu} \circ \Gamma \circ\left(w_{\mu}\right)^{-1}\right)$ and $T\left(w_{\nu} \circ \Gamma_{1} \circ\left(w_{\nu}\right)^{-1}\right)$.

Lemma 3.1 gives a bi-Lipschitz mapping between neighbourhoods of these two identity classes, and hence we have a bi-Lipschitz mapping between neighbourhoods of $[\mu] \in T(\Gamma)$ and $[\nu] \in T\left(\Gamma_{1}\right)$. 
Acknowledgements. This paper came about as a result of conjectures posed to the author by Vladimir Markovic, which have been resolved here. The author would like to thank Vladimir Markovic for the interesting discussions and support he has provided, and the referee for the helpful comments.

\title{
References
}

1. R. CoIfman and R. Rochberg, 'Representation theorems for Hardy spaces', Astérisque 77 (1980) 11-66.

2. F. GARDiner, Teichmüller theory and quadratic differentials (Wiley, New York, 1987)

3. F. Gardiner and N. LAKIC, Quasiconformal Teichmüller theory (American Mathematical Society, Providence, RI, 2000).

4. O. Lehto, Univalent functions and Teichmüller spaces (Springer, New York, 1987).

5. A. Pelczynski, 'Projections in certain Banach spaces', Studia Math. 19 (1960) 209-228.

6. J. Lindenstrauss and A. Pelczynski, 'Contributions to the theory of the classical Banach spaces', J. Funct. Anal. 8 (1971) 225-249.

7. M. Mateljević, 'The dual of the Bergman space defined on a hyperbolic plane domain', Publ. Inst. Math. (Beograd) (N.S.) 56(70) (1994) 135-139.

8. V. Markovic 'Biholomorphic maps between Teichmüller spaces', Duke Math. J. 120 (2003) 405-431.

9. W. Rudin, Real and complex analysis (McGraw-Hill, New York, 1987).

10. P. WojtaszczyK, ' $H_{p}$-spaces, $p \leqslant 1$, and spline systems', Studia Math. 77 (1984) 289-320.

\author{
Alastair Fletcher \\ Mathematics Institute \\ University of Warwick \\ Coventry, CV4 7AL \\ United Kingdom
}

fletcher@maths.warwick.ac.uk 